INGENIERÍA CIVIL

\title{
Estimación de los niveles de desempeño estructural y de la deriva inelástica de pórticos de concreto considerando la variabilidad de los materiales de Bogotá
}

CIVIL ENGINEERING

\section{Estimation of the structural performance levels and inelastic drift of reinforced concrete frames, considering the variability of the materials of Bogotá}

\author{
Daniel M. Ruiz ${ }^{* * \S}$, Maria C. Borrero ", María P. León ${ }^{* *}$, Hermes A. Vacca** \\ Departamento de Ingeniería Civil, ${ }^{* *}$ Grupo Estructuras, Pontificia Universidad Javeriana. \\ sborrero-m@javeriana.edu.co,sdaniel.ruiz@javeriana.edu.co,mpleon@javeriana.edu.co, \\ vacca@javeriana.edu.co
}

(Recibido: Junio 24 de 2011 -Aceptado: Abril 19 de 2012 -Versión Final: 22 Junio de 2012)

\begin{abstract}
Resumen
Los ingenieros estructurales calculan la capacidad sísmica de los pórticos de concreto reforzado con la geometría de los elementos estructurales y con las propiedades mecánicas de los materiales. Las propiedades mecánicas más importantes del concreto y el acero usadas para estimar dicha capacidad son: módulo de elasticidad (E) y resistencia a la compresión del concreto (f 'c); esfuerzo de fluencia (Fy) y esfuerzo resistente del acero (Fu); así como sus deformaciones unitarias. Basados en lo anterior se analizaron estadísticamente los resultados de más de 1,500 ensayos de compresión de concretos (solamente para Bogotá) y más de 900 pruebas de tensión de varillas. Luego se modelaron (análisis por elementos finitos tipo "frame” o barra) y diseñaron edificaciones aporticadas de concreto reforzado de 5 pisos de acuerdo con el reglamento colombiano de construcción sismo resistente (NSR10); y usando dos de los espectros de la microzonificación de Bogotá (año 2010). Usando los modelos de pórticos planos correspondientes a uno de los ejes de la edificación y con la generación de números aleatorios de las propiedades mecánicas del acero y el concreto (de acuerdo con los análisis estadísticos de los ensayos de laboratorio) se realizaron 80,000 análisis de pushover de los pórticos planos seleccionados con el fin de establecer las derivas inelásticas y sus funciones de distribución de probabilidad para diferentes niveles de daño (simulaciones de Montecarlo). De acuerdo con los resultados, las derivas promedio para alcanzar niveles de daño de fluencia, ocupación inmediata y protección a la vida son $0.51 \%, 0.84 \%$ y $1.41 \%$ respectivamente.
\end{abstract}

Palabras Claves: Deriva inelástica, Simulaciones de Montecarlo, Análisis de Pushover, Análisis estadísticos, Ensayos de laboratorio.

\begin{abstract}
The structural engineers calculate the seismic capacity of reinforced concrete frames with the geometry of the structural elements and with the mechanical properties of the materials. The most important mechanical properties of the concrete and the steel used to estimate this capacity are: elasticity modulus (E) and compressive strength ( $\mathrm{f}$ ' $c$ ) of the concrete, yield stress (Fy) and maximum stress of the steel $(\mathrm{Fu})$ and its strains. Based on these facts, results of more than 1,500 concrete compression tests (only from Bogotá), and more than 900 steel tension tests were statistically studied. Then, five stories reinforced concrete buildings were modeled (finite element analysis - frame elements) and designed according to the Colombian Earthquake Resistant Code (NSR10 ) and with two design spectrums of the Bogota microzoning (2010). Using the frame models and generating random numbers of the steel and concrete mechanical properties (according to the statistic analyses of the laboratory test) 80,000 pushover analyses of 2D frames were performed to obtain the inelastic drift and its probability distribution functions for different damage levels (Montecarlo simulations). According to the results the average drifts limits for a yield damage level, immediate occupancy damage level and life safety damage level are $0,51 \%, 0,84 \%$ and $1,41 \%$ respectivel.
\end{abstract}

Keywords: Inelastic drift, Montecarlo simulations, Pushover analysis, Statistic analyses of laboratory tests 


\section{Introducción}

El comportamiento sísmico de las edificaciones de concreto reforzado depende entre otros aspectos, de dos variables fundamentales: su curva de capacidad (fuerza vs. desplazamiento en los rangos elástico e inelástico) y los movimientos del terreno (efectos externos). Sobre esta segunda variable no se tiene hasta la actualidad ningún tipo de control ya que obedece a fenómenos que dependen del comportamiento de las capas superficiales de la corteza terrestre. Sin embargo las incertidumbres sobre las curvas de capacidad (en fuerza y desplazamiento) depende de variables ampliamente conocidas: las dimensiones de los elementos estructurales, cimentación, las cargas actuantes en la historia de la estructura y las características mecánicas de los materiales (resistencia y flexibilidad). De acuerdo con Prieto et. al. (2005) el conocimiento de las incertidumbres involucradas en el proceso de estimación del punto de desempeño estructural es esencial tanto para el diseño como para la estimación de riesgo y pérdidas esperadas por futuros sismos. De acuerdo con González e.t al. (2005) las diferencias entre los resultados analíticos y experimentales de respuestas de edificios sometidos a eventos sísmicos, son ocasionadas por las incertidumbres involucradas en el proceso de diseño, que hacen referencia a la variación entre la resistencia real y nominal de los materiales, las diferencias entre las solicitaciones reales y las empleadas en el diseño, y el grado de aproximación entre el modelo propuesto y el construido. La estimación de estas incertidumbres no se realiza frecuentemente en el caso colombiano. No obstante en las referencias Prieto et. al. (2005) y Muñoz et. al. (2005) se realizaron estimaciones de los desplazamientos espectrales en el punto de desempeño y se asociaron dichos desplazamientos a niveles de daño. En la Figura 1 se presentan las funciones de distribución de probabilidad y las funciones acumuladas reportadas por la referencia Prieto et. al. (2005), en donde se puede advertir agrupamientos de los datos en torno a valores medios relativamente bien definidos. Dichos agrupamientos se observan como rampas en la función de distribución acumulada. En Prieto et. al. (2005) se definieron niveles de daño para una edificación indispensable (un hospital), en donde la denominación de los diferentes estados de daño se propusieron semejantes a los determinados en FEMA/NIBS (1999), (Leve, Moderado, Extenso y Completo).

\section{Metodología}

Con base en lo anterior se ha establecido una metodología para estimar la incertidumbre aleatoria de la deriva inelástica y de los niveles de daño de edificaciones de 5 pisos; diseñadas en Bogotá con el reglamento colombiano de construcción sismo resistente, AIS (2010), y con dos de los espectros de la nueva microzonificación presentada en FOPAE (2010). Dicha metodología se describe a continuación:

Determinación de las funciones de distribución de probabilidad de las principales propiedades mecánicas (usadas en el diseño estructural) del acero y el concreto en Bogotá: resistencia a la compresión del concreto $\left(f f^{`} c\right)$, módulo de elasticidad del concreto $(E)$, esfuerzo de fluencia del acero $(F y)$, esfuerzo máximo resistente del acero $(F u)$ y la deformación máxima de rotura $\left(\varepsilon_{M A X}\right)$.

Diseño de dos edificaciones aporticadas de 5 pisos mediante dos espectros de diseño de la microzonificación sísmica presentada en FOPAE (2010). Se usó un espectro tipo "Piedemonte" y otro tipo "Lacustre". Las propiedades de los materiales fueron: $f^{\prime} c=28 \mathrm{MPa}, F y=420 \mathrm{MPa}$, $4,700 \sqrt{f^{\prime \prime} c}=24,870$ MPa y $\gamma_{\text {concreto }}=24 \mathrm{kN} / \mathrm{m}^{3}$.

Generación de variables aleatorias para $f^{\prime} c, E, F y$ y para $F u$ según las funciones de distribución de probabilidad establecidas en el apartado 1 .

La incertidumbre aleatoria en la curva de capacidad se estima por medio de simulaciones de Montecarlo para análisis estáticos no lineales de "pushover" de los modelos estructurales de los dos pórticos planos de concreto reforzados. Se realizaron 80,000 simulaciones de Montecarlo, 40,000 por pórtico plano. 


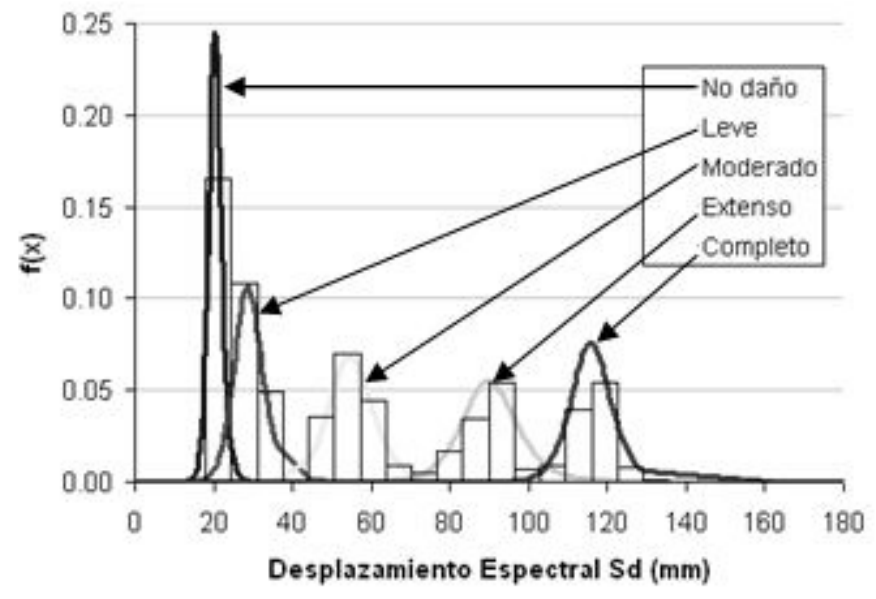

a)

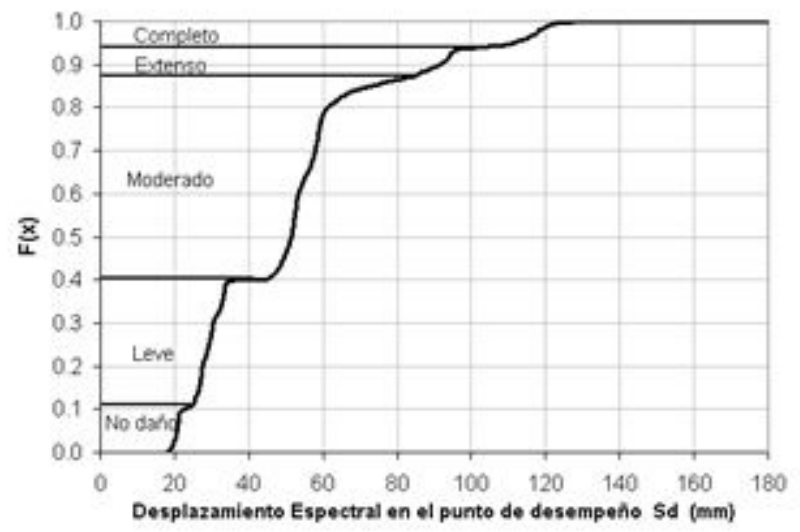

b)

Figura 1. a) Agrupamiento de los puntos de desempeño de la edificación esencial en estados de daño; b) Propuesta de diferentes niveles de estado de daño. Tomada de Prieto et. al. (2005).

Para cada una de las 80,000 curvas de capacidad se determinaron las derivas (en porcentaje de la altura de entrepiso) de todos los niveles de las dos edificaciones.

Se buscaron posteriormente las derivas máximas de entrepiso necesarias para que se empezaran a presentar los niveles de daño en los términos establecidos en la referencia FEMA356 (2000). Son de particular importancia para el presente estudio los niveles de daño de fluencia (B), ocupación inmediata (IO) y protección a la vida (LS).
En el presente estudio se tratarán únicamente las propiedades mecánicas de los materiales como variables aleatorias, manteniendo constantes y como variables determinísticas las dimensiones de los elementos estructurales. La segunda fase de la investigación, en la que se considera la aleatoriedad de las características dimensionales, está siendo objeto de estudio actualmente por parte del grupo de investigación Estructuras y Construcción de la Pontificia Universidad Javeriana. Se ha realizado esta división de las variables geométricas y mecánicas con el fin de determinar la influencia que tiene cada una en el 
comportamiento probabilístico global de la estructura a nivel de derivas.

\section{Resultados y discusión}

\subsection{Variabilidad de las propiedades mecánicas del concreto y el acero para Bogotá}

Las propiedades de los materiales no tienen valores exactos, razón por la cual siempre existe dispersión y variabilidad. Sin embargo, existen pocos estudios en los que se relacione la variabilidad de los materiales con el diseño de estructuras de concreto reforzado en Colombia.

Con base en lo anterior se recopilaron, únicamente para la ciudad de Bogotá, resultados de ensayos compresión de concreto y tensión de acero. La base de datos contiene resultados de 1566 ensayos a compresión para la determinación simultánea de $f$ ' $c$ y $E$ y 986 ensayos de los que se determinó $F y, F u$ y $\varepsilon_{M A X}$ en el acero. Los ensayos de compresión fueron hechos como lo estipula la norma NTC4025 (2005) y los ensayos de tensión de varillas de acero se hicieron siguiendo la norma NTC2289 (2005). Los ensayos de compresión se hicieron sobre cilindros estándar de obras nuevas así como sobre núcleos extraídos de edificaciones existentes. La gran mayoría de los resultados disponibles se tomaron de los reportes de ensayos realizados entre los años 2004 y 2010 por el Laboratorio de Pruebas y Ensayos del Departamento de Ingeniería Civil de la Pontificia Universidad Javeriana. Cabe anotar que desde antes del año 2005, fecha en la que se implementó la norma NTC4025, el Laboratorio de Pruebas y Ensayos hacía uso de las normas ASTM, las cuales sirvieron de base a las normas NTC. Es importante mencionar que estos dos ensayos están incluidos dentro de la resolución de acreditación que el Organismo Nacional de Acreditación de Colombia le dio a este Laboratorio en el año 2010 (09-Lab-023). Así mismo el laboratorio tuvo una acreditación desde el año 2004 hasta el año 2009 por la Superintendencia de Industria y Comercio (resolución 16395). En ese orden de ideas los ensayos de laboratorio se hicieron con todos los estándares de calidad como se especifica en la norma ISO 17025 (2005). Esto sugiere que los ensayos experimentales fueron hechos con equipos de alta resolución y con todos los certificados de calibración respectivos.

En las Figuras $2 \mathrm{a}$ y $2 \mathrm{~b}$ se presentan los datos estadísticos para $f$ ' $c$ y para $E$. En dichas gráficas se muestra también la función de distribución de probabilidad que más se ajusta a las variables estudiadas Para la determinación de dichas distribuciones se usó la técnica de bondad del ajuste de Kolmogorov-Smirnov, de acuerdo con la referencia Canavos (1986). Esta prueba parte de un estadístico de contraste entre las dos siguientes hipótesis:

$\mathrm{H}_{0}$ : Los datos analizados siguen una distribución $\mathrm{X}$

$\mathrm{H}_{1}$ : Los datos analizados no siguen una distribución X

El estadístico de contraste es el siguiente:

$D=\underset{1 \leq i \leq n}{S U P}\left|\bar{F}_{n}\left(x_{i}\right)-F_{0}\left(x_{i}\right)\right|$ en donde:

$x_{i}$ es el i-ésimo valor observado en la muestra (cuyos valores se han ordenado previamente de menor a mayor).

$\bar{F}_{n}\left(x_{i}\right)$ es un estimador de la probabilidad de observar valores menores o iguales que $x_{i}$

$F_{0}\left(x_{i}\right)$ es la probabilidad de observar valores menores o iguales que $x_{i}$ cuando $\mathrm{H}_{0}$ es cierta.

Así pues, D es la mayor diferencia absoluta observada entre la frecuencia acumulada observada $\bar{F}_{n}\left(x_{i}\right)$ la frecuencia acumulada teórica $F_{0}\left(x_{i}\right)$, obtenida a partir de la distribución de probabilidad que se especifica como hipótesis nula.

Si los valores observados son similares a los esperados $F_{0}\left(x_{i}\right)$, el valor de $D$ será pequeño.

Cuanto mayor sea la discrepancia entre la distribución empírica $F_{n}\left(x_{i}\right)$ y la distribución teórica, mayor será el valor de $D$. Por tanto, el criterio para la toma de la decisión entre las dos hipótesis será de la forma: 


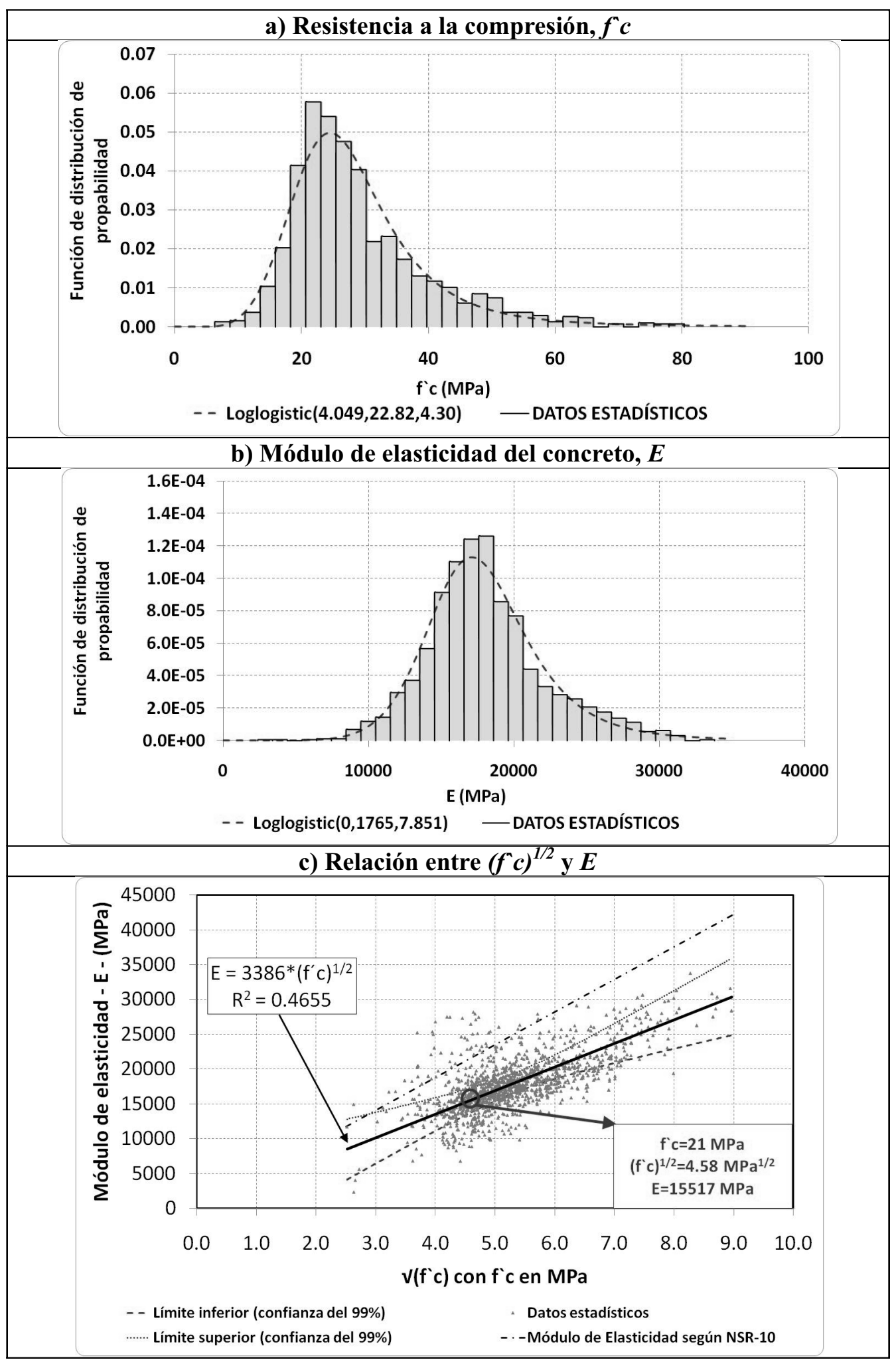

Figura 2. a)Distribución ajustada a los datos de $f^{~ ' c ~ b) ~ D i s t r i b u c i o ́ n ~ a j u s t a d a ~ a ~ l o s ~ d a t o s ~ d e ~ E ~}$ c) Regresión estadística para $E$ en función de $\sqrt{f^{\circ} c}$ 
Si $\mathrm{D} \leqslant \mathrm{D} \alpha$ entonces se acepta $\mathrm{H}_{0}$ Si $\mathrm{D} \geqslant \mathrm{D} \alpha$ entonces se rechaza $\mathrm{H}_{0}$

En las anteriores expresiones $\alpha$ corresponde al nivel de significación del contraste.

En la Tabla 1 se muestran las principales características tanto de las funciones de ajuste como de la muestra estadística. Obsérvese que los coeficientes de variación de la resistencia a la compresión es muy alta mostrando que a pesar de que existan concretos típicos de diseño de $21 \mathrm{MPa}$ y $28 \mathrm{MPa}$ las edificaciones en Bogotá tiene resistencias a la compresión muy variables. Es claro que la variabilidad de los módulos de elasticidad es mucho menor aunque consistentemente alta. En la mayoría de los casos las pruebas de laboratorio se hacen sin el conocimiento de las resistencias de diseño, como parte de los requisitos de conflicto de intereses de la norma de acreditación de laboratorios, ISO EIC 17025.

Para el presente estudio se utilizó como f 'c de diseño el valor medio de los datos estadísticos, el cual está en el orden de magnitud de los concretos de $28 \mathrm{MPa}$. Desde el punto de vista del diseño en concreto reforzado, el momento resistente de una sección depende prioritariamente de la cantidad de acero y de la altura de la sección; siendo menos importante la variable f 'c. Por ejemplo, si en una sección de concreto reforzado se mantienen constantes las variables geométricas y las propiedades mecánicas del acero; y se disminuye $\mathrm{f}$ 'c de $28 \mathrm{MPa}$ a $21 \mathrm{MPa}$, el momento resistente disminuirá tan solo en un $5 \%$ y la rigidez de la sección en un $15 \%$. Los cambios de f'c afectan en un mayor porcentaje las cargas axiales resistentes; las cuales no son de marcada importancia en edificios de pocos pisos. Bajo este punto de vista; y si lo que pretende el estudio es dar una mirada general al comportamiento global de una edificación de 5 pisos, el tener concretos de diferentes resistencias de diseño en la misma base de datos no es una limitante que cambie las tendencias de los resultados en un porcentaje superior al 15\%. Bajo el mismo razonamiento, la diferencia normalmente aceptada (y demostrada) entre los resultados que arrojan los ensayos de compresión de cilindros de concreto y los ensayos de compresión de núcleos extraídos (ambos incluidos en la base de datos) no cambiarán las tendencias de los resultados; aunque claramente inducen una limitación al estudio.

Por su parte en la Figura 2c se muestra la línea de ajuste que relaciona el módulo de elasticidad (ordenadas) con la raíz cuadrada de la resistencia a la compresión con su respectivo $\mathrm{R}^{2}$ el cual es de 0.46. Simultáneamente se muestran las líneas que definen el intervalo de confianza (al $99 \%$ ) de la ecuación de ajuste por mínimos cuadrados. Obsérvese también que la curva sugerida por la normativa vigente $\left(E=4,700 \sqrt{\mathrm{f}^{\prime} \mathrm{c}}\right.$, está muy por encima de la ecuación de ajuste para los concretos bogotanos que se observa en la figura, ya que el coeficiente que multiplica a $\sqrt{\mathrm{f}^{\circ} \mathrm{c}}(4,700 \mathrm{en} \mathrm{MPa})$ es un $39 \%$ superior al valor de la regresión

Tabla 1. Datos estadísticos de las variables $E$ y $f^{\prime}{ }^{\prime} c$

\begin{tabular}{ccccc}
\hline & \multicolumn{2}{c}{$E$} & \multicolumn{2}{c}{$f^{\prime c} c$} \\
\hline $\begin{array}{c}\text { Tipo de distribución } \\
\text { ajustada }\end{array}$ & \multicolumn{2}{c}{ Loglogistic(0,1765,7.851) } & \multicolumn{2}{c}{ Loglogistic(4.049,22.82,4.30) } \\
\hline Parámetro & Datos & Distribución & Datos & Distribución \\
\hline Mínimo & $2,373.1 \mathrm{MPa}$ & 0 & $6.4 \mathrm{MPa}$ & 4.0 \\
Máximo & $33,770.7 \mathrm{MPa}$ & Infinito & $80.3 \mathrm{MPa}$ & Infinito \\
Media & $18,073.4 \mathrm{MPa}$ & $18,129.7 \mathrm{MPa}$ & $29.2 \mathrm{MPa}$ & $29.0 \mathrm{MPa}$ \\
Coefíciente de & $23 \%$ & $24 \%$ & $38 \%$ & $41 \%$ \\
variación & 0.5 & 1.3 & 1.4 & 3.5 \\
asimetría & 3.8 & 8.6 & 5.5 & 107.3 \\
Kurtosis & 1,566 & ---- & 1,566 & --- \\
no de datos & & & & \\
\hline
\end{tabular}


estadística (3,386 en $\mathrm{MPa})$. Este comportamiento (pero con menos muestras estadísticas) ya se había anotado en la referencia Ruiz et. al. (2007).

Por su parte en la Figura 3 se muestran los datos estadísticos procesados para $F y$, para $F u$ y para $\varepsilon_{M A X}$. En las gráficas se muestra también la función de distribución de probabilidad que más se ajusta. Al igual que para el caso del concreto se usó la técnica de Kolmogorov-Smirnov. En la Tabla 2 se muestran las principales características tanto de las funciones de ajuste como de la muestra estadística para estas tres variables que caracterizan al acero.

A nivel nacional es difícil encontrar artículos científicos en los que se estudie de manera detallada las características de los materiales de construcción colombianos. Sin embargo afortunadamente en González et. al. (2005) se presentan más de 460 ensayos de tensión de varillas de diferentes siderúrgicas del país. En dicha referencia los valores medios de $F y, F u$ y $\varepsilon_{M A X}$ son respectivamente $470 \mathrm{MPa}, 660 \mathrm{MPa}$ y $14.1 \%$. Los valores de esfuerzo son altos comparativamente con los obtenidos en la Tabla 2 y la deformación unitaria máxima reportada en esta referencia es muy baja al compararla con el valor medio de la misma tabla.

Se anota que los valores de la Tabla 2 están más cerca de las especificaciones de la Norma NTC2289 (2005) que los valores reportados en González et. al. (2005). Por otro lado se observa que los coeficientes de variación de Fy, Fu y $\varepsilon_{\mathrm{MAX}}$ reportados por González et. al. (2005) (5.4\%, 4.6\% y $12.5 \%$ respectivamente) son muy similares a los de la Tabla 2. Debe anotarse que existen dentro de la base de datos aceros cuya especificación era de 420 $\mathrm{MPa}$ pero que tenían un origen dudoso. Estas son muy pocas barras de acero en la base de datos (11 de un total de 986) pero que deben incluirse como posibles aceros usados en algunas edificaciones de la ciudad. Claramente estas barras no tienen una influencia importante sobre la base de datos ni sobre los parámetros estadísticos de las propiedades mecánicas (media y desviación estándar). Finalmente, para ser usado en las simulaciones de Montecarlo, se hizo un análisis estadístico para la relación $\mathrm{Fu} / \mathrm{Fy}$ que arrojó un valor medio de 1.48 y un coeficiente de variación de $4.7 \%$.
Es claro que los coeficientes de variación de las propiedades mecánicas del acero son muy inferiores a las del concreto lo cual está asociado a procesos de control de calidad durante la construcción de las edificaciones y durante la fabricación de los cilindros de ensayo así como en la especificación del concreto para las obras civiles.

\subsection{Edificaciones a analizar}

Las edificaciones diseñadas en esta investigación obedecen a una planta típica de una estructura estudiada en los años 90 para hacer los balances económicos de las edificaciones diseñadas con la norma AIS (1998), NSR-98 (Véase la Figura 4). Este estudio económico se puede apreciar en García (1996). Las edificaciones de planta constante para comparación, son simétricas, no tienen irregularidades en altura ni en planta, no tienen reducción de secciones con la altura, cambios de materiales o sus propiedades y tampoco defectos como columnas cortas o pisos bajos débiles. Corresponde a edificaciones ideales que en la realidad son difíciles encontrar, pero que se usaron en esta investigación para fines de comparación y evaluación. Se realizó el diseño del pórtico tridimensional y se usó para el análisis del presente trabajo el pórtico plano del eje 3. Dicho pórtico tiene 5 pisos, con luces de 9 metros y altura de entrepiso de $3 \mathrm{~m}$ como se muestra en la Figura 5. Las características de los materiales usados fueron extraídos de la referencia AIS (2010): $f$ ${ }^{\prime} c=28 \mathrm{MPa}, F y=420 \mathrm{Mpa}, \quad 4,700 \sqrt{f^{\prime} c}=24,870$ MPa y $\gamma_{\text {concreto }}=24 \mathrm{kN} / \mathrm{m}^{3}$. Para realizar el análisis, se tomaron dos casos extremos de respuesta local del suelo de acuerdo con la referencia FOPAE (2010) a la luz de la nueva microzonificación sísmica de Bogotá: Piedemonte B y Lacustre-500. Los espectros de estas zonas se muestran en la Figura 6. La carga muerta de diseño fue de $7.78 \mathrm{kN} / \mathrm{m}^{2}$ (que incluye el entrepiso y la carga de muros, entre otros) a excepción de la cubierta diseñada con 3.34 $\mathrm{kN} / \mathrm{m}^{2}$. La carga viva de diseño fue de $1.8 \mathrm{kN} / \mathrm{m}^{2}$ (carga de edificaciones de vivienda).

Con base en las anteriores características se establecieron las dimensiones de elementos estructurales (véase la Figura 5) que garantizan el cumplimiento de los requisitos de resistencia y 


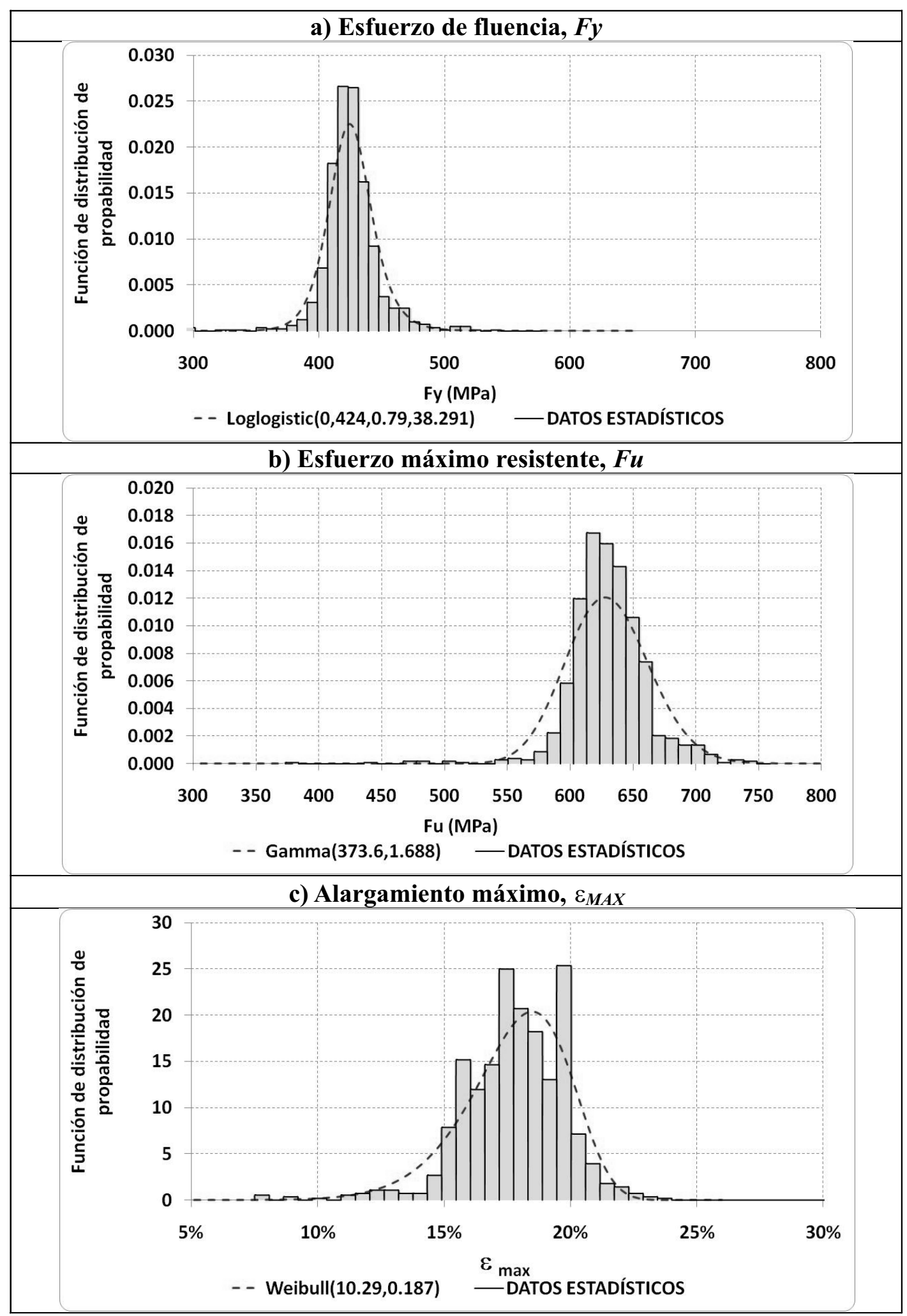

Figura 3. a)Distribución ajustada a los datos de Fy b) Distribución ajustada a los datos de Fu c) Distribución ajustada a los datos de $\varepsilon_{\operatorname{MAx}}$ 
flexibilidad (deriva de diseño $=0.9 \%$ para ambos edificios) de la NSR-10. El diseño se hizo con base en la Fuerza Horizontal Equivalente.

\subsection{Análisis de Pushover}

La metodología Pushover permite estimar la demanda sísmica y verificar el desempeño de una estructura ante movimientos del terreno. El análisis de pushover se basa en procedimientos estáticos no-lineales con distribuciones de fuerzas laterales que incluye las contribuciones de los modos de vibración del comportamiento elástico mediante la teoría clásica de la dinámica de estructuras. A partir del análisis realizado al sistema de varios grados de libertad (edificio de varios pisos) se obtienen las curvas del desplazamiento de la cubierta vs. el cortante basal hasta un desplazamiento lateral máximo esperado,

Tabla 2. Datos estadísticos de las variables $F y, F u$ y $\varepsilon_{M A X}$

\begin{tabular}{|c|c|c|c|c|c|c|}
\hline \multirow{3}{*}{$\begin{array}{c}\text { Tipo de } \\
\text { distribución } \\
\text { ajustada }\end{array}$} & \multicolumn{2}{|c|}{$F y$} & \multicolumn{2}{|c|}{$F u$} & \multicolumn{2}{|c|}{$\varepsilon_{M A X}$} \\
\hline & \multicolumn{2}{|c|}{$\begin{array}{c}\text { Loglogistic } \\
(0,424,0.79,38.291)\end{array}$} & \multicolumn{2}{|c|}{$\begin{array}{c}\text { Gamma } \\
(373.6,1.688)\end{array}$} & \multicolumn{2}{|c|}{$\begin{array}{c}\text { Weibull } \\
(10.29,0.187)\end{array}$} \\
\hline & Datos & Distribución & Datos & Distribución & Datos & Distribución \\
\hline Mínimo & $377 \mathrm{MPa}$ & $0.0 \mathrm{MPa}$ & $373.4 \mathrm{MPa}$ & 0.0 & $7.5 \%$ & $0 \%$ \\
\hline Máximo & $602.3 \mathrm{MPa}$ & & $790.3 \mathrm{MPa}$ & $\infty$ & $24.0 \%$ & $\infty$ \\
\hline Media & $425.7 \mathrm{MPa}$ & $425.3 \mathrm{MPa}$ & $630.6 \mathrm{MPa}$ & $630.6 \mathrm{MPa}$ & $17.8 \%$ & $18 \%$ \\
\hline $\begin{array}{l}\text { Coeficiente de } \\
\text { variación }\end{array}$ & $5.76 \%$ & $5.75 \%$ & $5.07 \%$ & $5.17 \%$ & $11.24 \%$ & $11.11 \%$ \\
\hline Asimetría & 0.2 & 0.2 & -0.6 & 0.1 & -0.8 & -0.6 \\
\hline Kurtosis & 14.6 & 4.3 & 12.2 & 3.0 & 5.7 & 3.6 \\
\hline No de datos & 986 & ---- & 986 & ---- & 986 & ---- \\
\hline
\end{tabular}

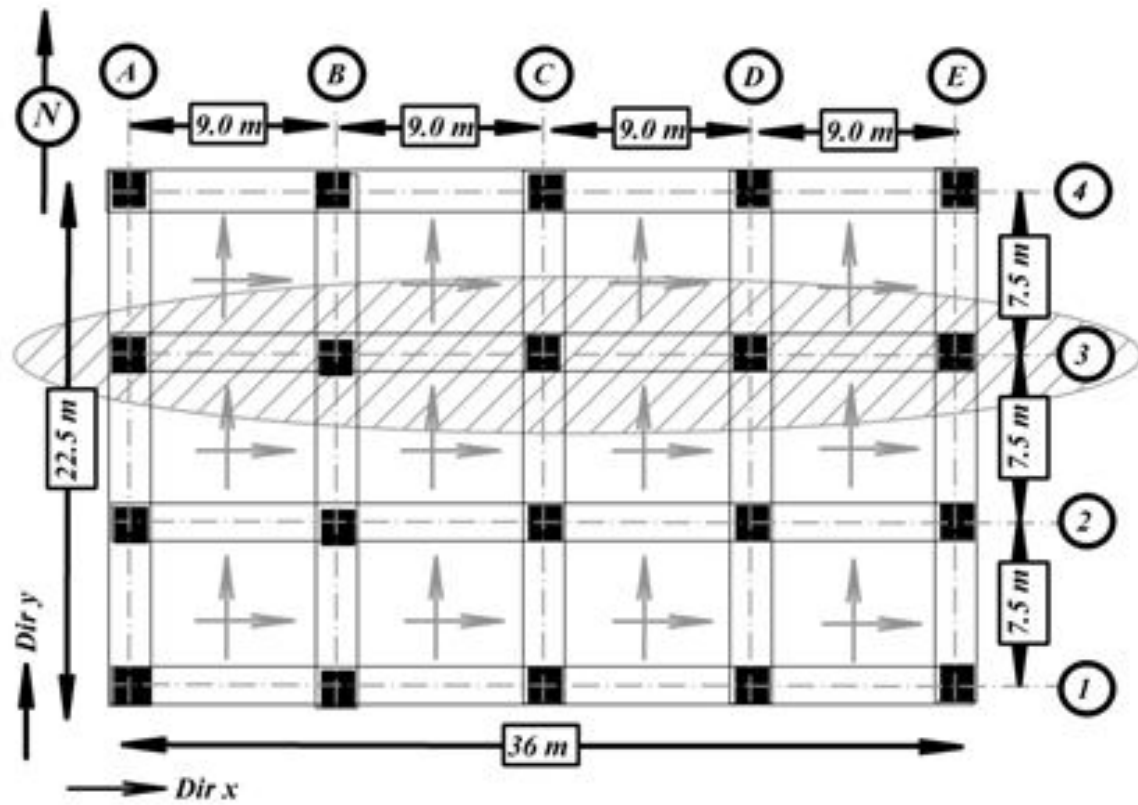

Figura 4. Planta Típica adaptada de García (1996) 
usando distribuciones de fuerzas laterales proporcionales a las formas modales. Como resultado del análisis se obtiene también información del comportamiento no-lineal de la edificación como la secuencia de fluencia y falla (las secciones van ingresando al rango no-lineal), los desplazamientos laterales en cada incremento de carga, la deriva de entrepisos, las fallas de elementos por flexión y/o corte etc.

Para realizar el análisis no lineal estático de Pushover, es necesario asignar a las vigas y a las columnas los diagramas momento curvatura. Adicionalmente a las columnas se les debe asignar

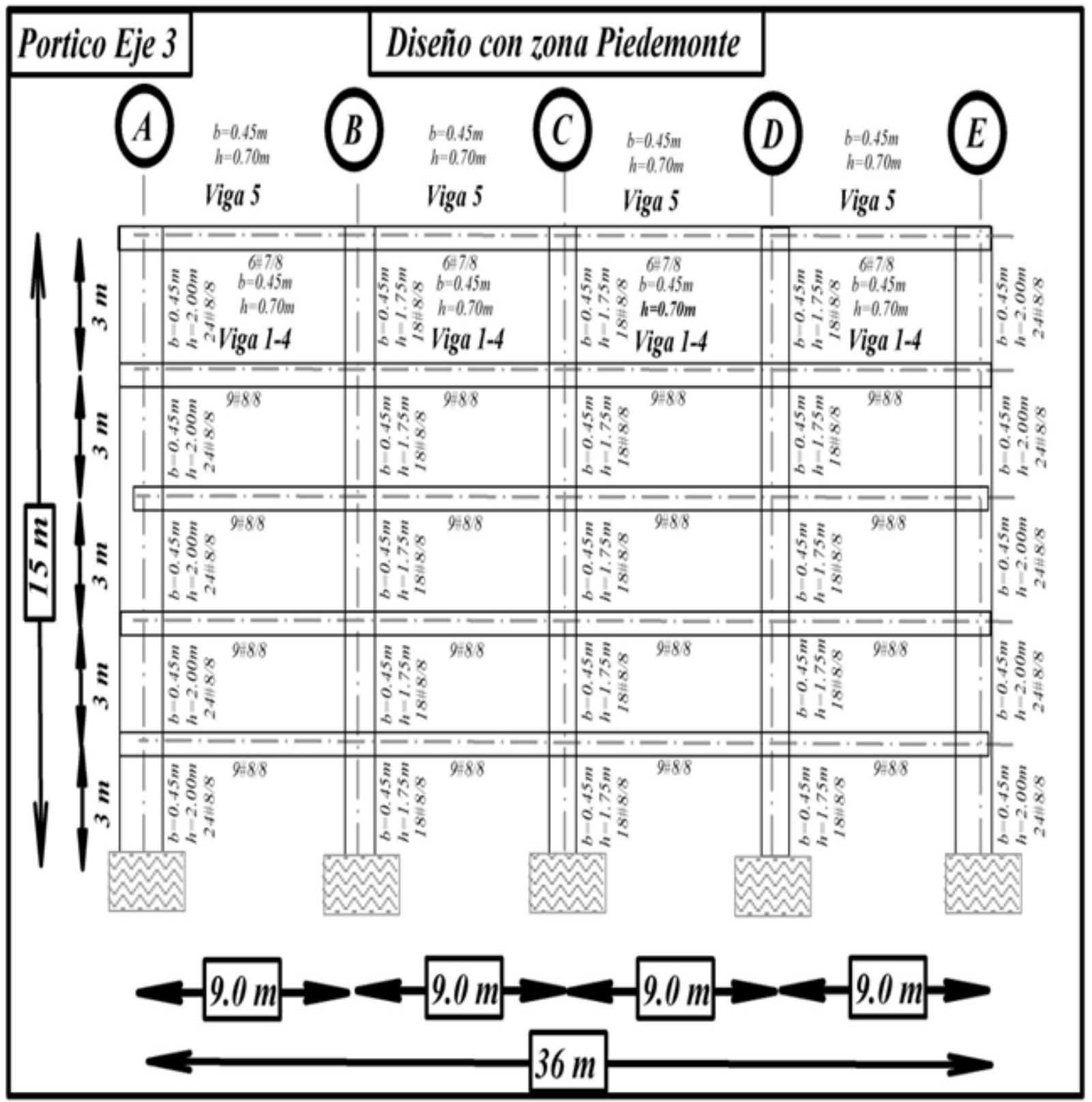

a) 


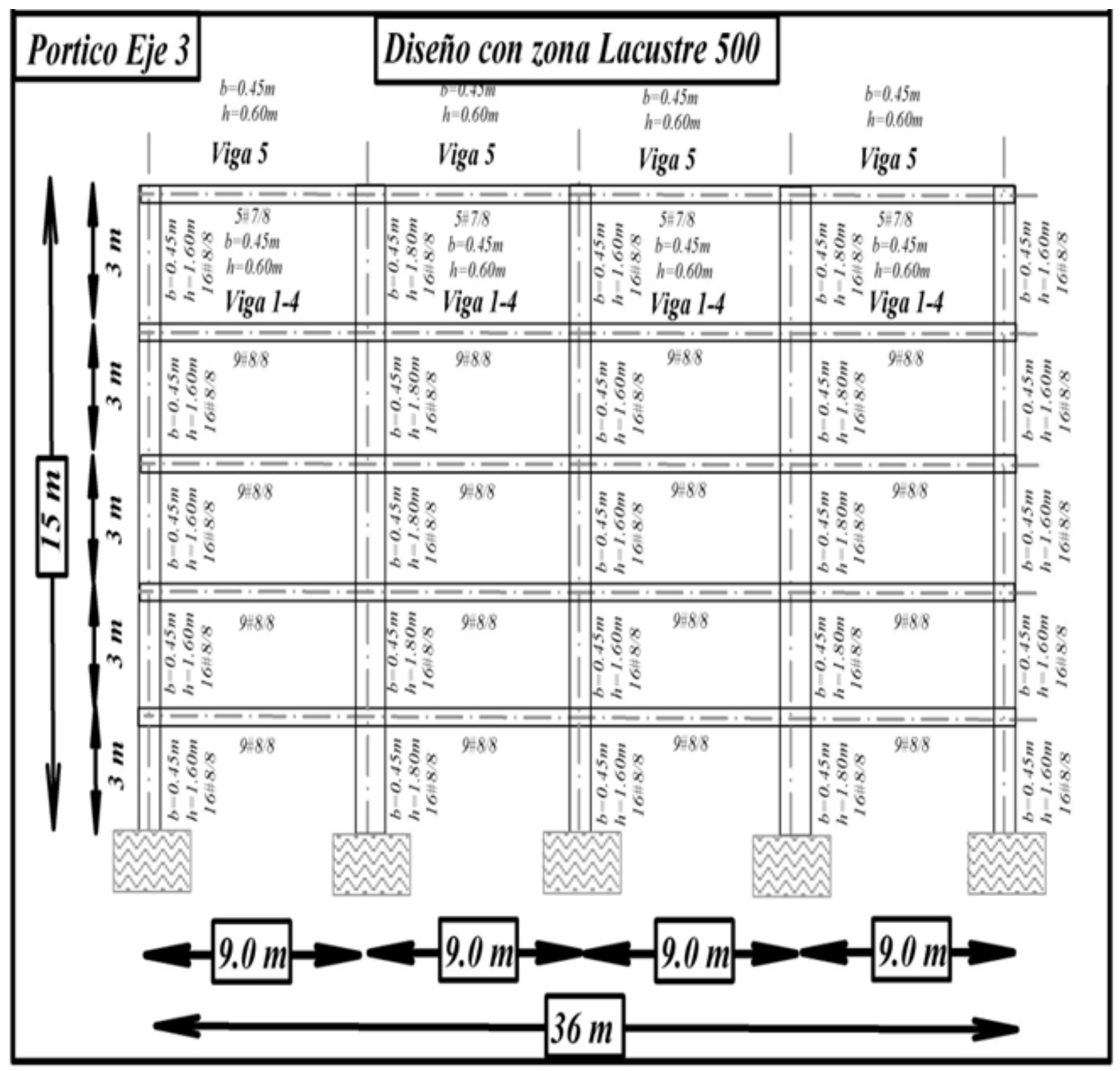

b)

Figura 5.Pórtico eje 3 a) Diseño espectro Piedemonte B b)Diseño espectro Lacustre500

el diagrama de interacción. Se debe aclarar que se definieron e incluyeron al menos 10 diagramas momento curvatura para diferentes porcentajes de la carga axial máxima resistente, para cada una de las secciones transversales; todo esto mediante diferentes programas elaborados por los autores en Microsoft Excel®. Ya que los valores de los diagramas momento curvatura y el diagrama de interacción se determinan con las características de los materiales y de las secciones, cada vez que se modifica $f^{\text {' }} \mathrm{C}, E, F y$ y $F u$ se debía modificar el diagrama de interacción y el de momentocurvatura y el diagrama de momento-rotación; todo esto en función de la carga axial de las diferentes combinaciones de carga para las columnas. Ejemplos de un diagrama de interacción y de un diagrama de momento-rotación de las edificaciones analizadas 


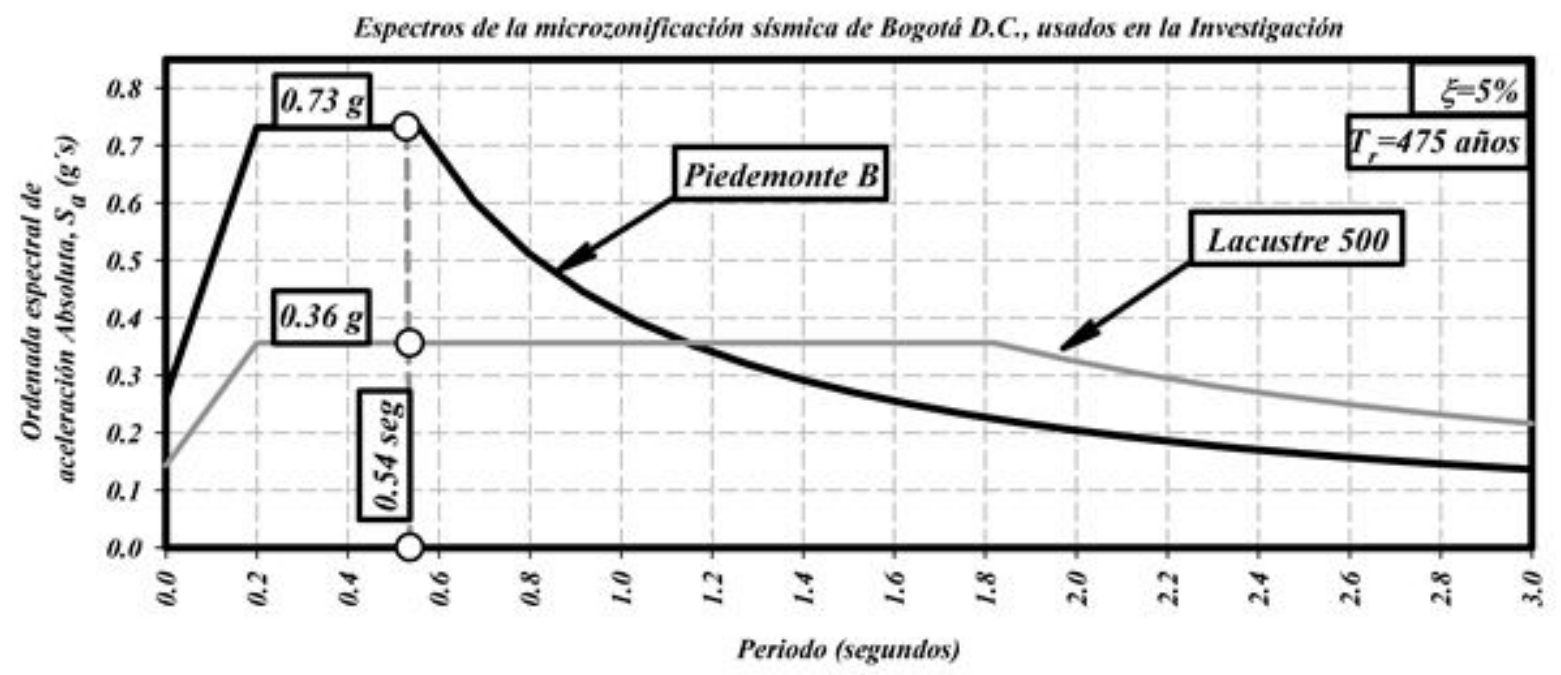

Figura 6. Espectros de diseño usados

se presenta en la Figura 7. Es importante aclarar que el diseño original se realizó con las propiedades especificadas de los materiales (indicadas en el resumen de la metodología utilizada), por lo tanto la sección y la cantidad de refuerzo es única para cada elemento. Se verificó en todos los casos que los elementos no fluyeran para la condición de carga gravitacional; y que de esta manera la fluencia ocurriera únicamente por la condición de carga sísmica. Así mismo se verificó que en la totalidad de los casos la fluencia por flexión ocurriera antes de la falla por cortante.

Con los parámetros adecuados, el programa SAP2000, CSI (2007), está en capacidad de inducir esfuerzos, fuerzas y momentos generados por incrementos en el desplazamiento de la estructura, de tal forma que se puede observar cómo se degrada la rigidez de cada elemento en función de sus características no lineales. La variación del comportamiento de los elementos es monitoreada por el programa a través de diferentes niveles de desempeño como se establece en la referencia FEMA356 (2000). Dichos niveles de daño se muestran en la Figura 8, en donde es de particular importancia para el presente estudio los niveles de daño de fluencia $(B)$, ocupación inmediata $(I O)$ y protección a la vida (LS). Es importante mencionar que el cálculo de cada estado límite de cada uno de los elementos

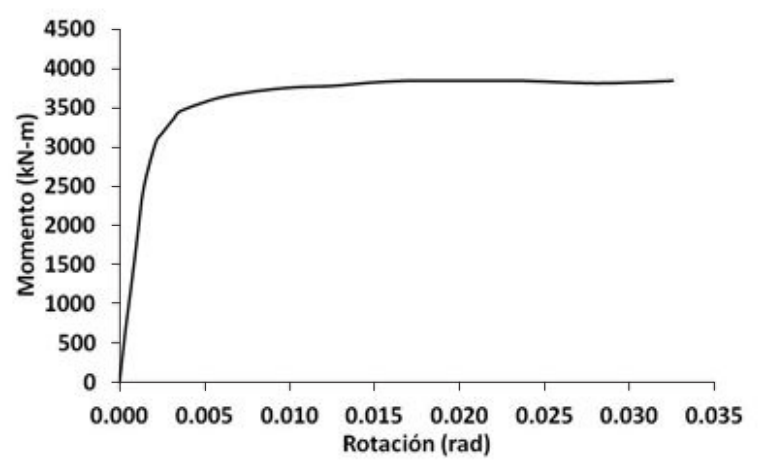

a)

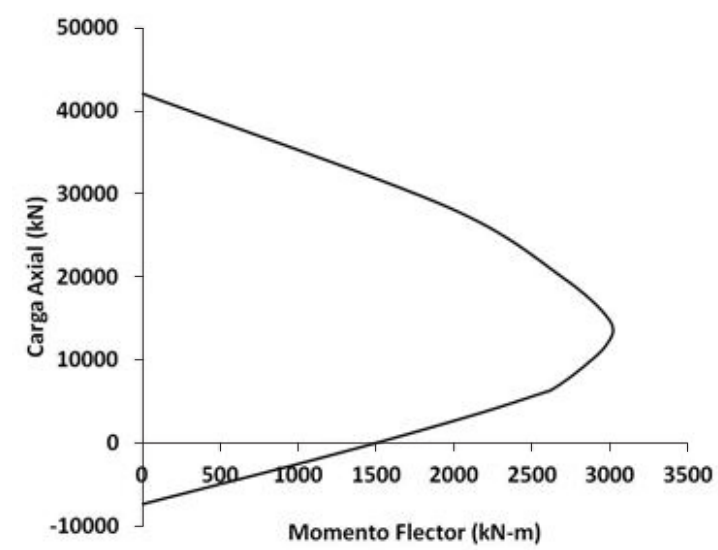

b)

Figura 7. a) Ejemplo de diagrama momento rotación y b) Ejemplo de diagrama de interacción. 
se calculó manualmente siguiendo las especificaciones y recomendaciones del FEMA mediante programas desarrollados en Visual Basic para Excel ${ }^{\circledR}$. El $I O$ se asocia con un estado de daño posterremoto en que apenas se presenta daño estructural muy limitado. Los sistemas de resistencia de fuerzas verticales y horizontales mantienen prácticamente sus características y capacidades y los riesgos a la vida o daños personales por fallas $\in 1 / \sqrt{\mathrm{N}}$ urales son prácticamente descartables y la edificación es segura para ingresar, egresar u ocupar. Por su parte en el nivel de protección a la vida (LS) pueden haber ocurrido daños estructurales significativos pero se garantiza un margen suficiente contra el colapso total o parcial; aunque en este nivel de daño pueden ocurrir afectaciones a las personas durante el sismo, el riesgo de pérdida de vida por daños estructurales es relativamente bajo. Es importante anotar que edificios nuevos diseñados con la normativa sismorresistente vigente no deben alcanzar este nivel de daño.

Finalmente se debe anotar que la carga incremental del análisis de pushover se aplicó de manera proporcional a la masa de cada uno de los grados de libertad de los nudos del pórtico plano. De la misma forma el análisis no lineal estático se controló por los desplazamientos horizontales de los nudos del último piso.

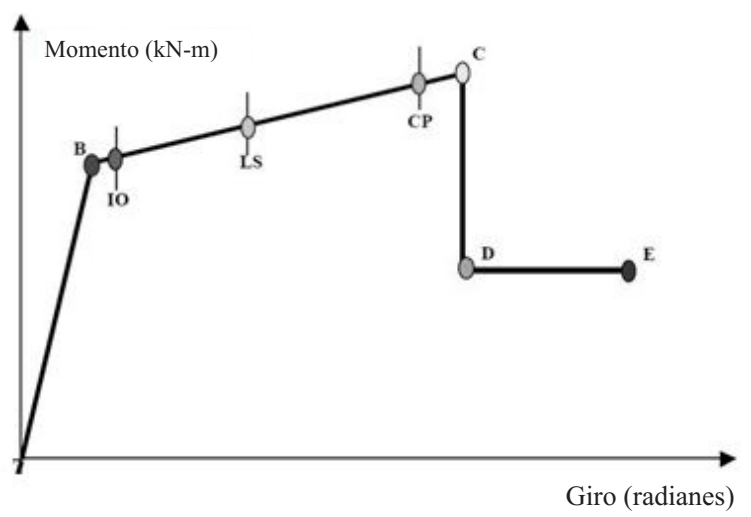

Figura 8. Niveles de daño FEMA356 (2000) y ATC40 (1996), $B$; Esfuerzo de fluencia de la sección, IO; Nivel de ocupación inmediata, LS; Nivel de protección a la vida, CP; Prevención del colapso, C; Rango de seguridad limitada, D; Estabilidad estructural, E; Colapso

\subsection{Simulaciones numéricas mediante el método de montecarlo}

El método de Montecarlo es un método no determinístico o estadístico numérico, usado para aproximar expresiones matemáticas complejas y costosas de evaluar con exactitud. El método de Montecarlo proporciona soluciones aproximadas a una gran variedad de problemas matemáticos posibilitando la realización de experimentos con muestreos de números pseudoaleatorios en un computador. El método es aplicable a cualquier tipo de problema, ya sea probabilístico o determinista. A diferencia de los métodos numéricos que se basan en evaluaciones en $\mathrm{N}$ puntos para producir una solución aproximada, el método de Montecarlo tiene un error absoluto de la estimación que decrece como en virtud del teorema del límite central. El método de Montecarlo consistió en este estudio en realizar simulaciones utilizando números aleatorios (generados en Excel ${ }^{\circledR}$ ), para determinar el comportamiento futuro de la variable aleatoria "deriva inelástica". El método se puede resumir en los siguientes pasos:

Especificar las variables y objetivos del modelo: geometría y propiedades mecánicas de los materiales.

Estimar la distribución de probabilidad que explica el comportamiento de las variables aleatorias no controladas del modelo: Las variables controladas fueron las geométricas y las no controladas fueron las propiedades mecánicas del acero y del concreto

Generar un número aleatorio con Excel para las propiedades mecánicas del concreto y del acero

Repetir el proceso un elevado número de veces hasta obtener la cantidad deseada de valores muestrales; en este caso valores de $F y$ y $F u$ combinados con valores de $f^{\prime} c$ y $E$.

Realizar con las variables obtenidas las operaciones especificadas en el modelo: esto implica la determinación de las rótulas plásticas y los diagramas de interacción de cada una de las secciones transversales y la ejecución de los análisis no lineales estáticos de "pushover". 
Por último analizar las funciones de distribución de las variables objetivo obtenidas con las operaciones indicadas; en este caso las derivas inelásticas y los niveles de daño.

Con base en la metodología de análisis de Montecarlo presentada anteriormente; y considerando las distribuciones estadísticas presentadas en párrafos anteriores para $f^{\prime} \mathrm{c}$ y $F y$, se generaron 200 números aleatorios para cada una de estas dos variables (400 en total). Las distribuciones de probabilidad de las dos variables principales estudiadas $\left(f^{\prime} c y F y\right)$ son LogLogistica, sin embargo, para la generación de los números aleatorios se consideró, para las dos variables, una distribución Normal. Lo anterior debido a que todos los programas se implementaron en Excel, desde donde se controlaban los programas de análisis de rótulas plásticas y diagramas de interacción así como SAP 2000. De acuerdo con González et. al. (2005) los valores que caracterizan la curva esfuerzo-deformación, se consideran variables continuas, por lo cual se puede buscar una aproximación de ajuste a una distribución normal. Por otro lado, de acuerdo con las referencias, Ang (1973) y Sánchez (2004) es posible realizar las simulaciones y análisis de confiabilidad aún para funciones de distribución de probabilidad diferentes a la normal con resultados satisfactorios siempre que cumplan con el teorema del límite central y cumplan con las características de una distribución Gaussiana (con una tendencia central y simétrica marcada).

Cada uno de los dos pórticos estudiados fue sometido a 40,000 análisis no lineales estáticos de Pushover. A cada uno de los análisis de pushover se asignó uno de los 200 valores simulados para $f^{\prime} c$ y uno de los 200 valores simulados para $F y$, lo que hacen un total de 80,000 simulaciones (200 valores $\operatorname{de} f^{\prime} c$ x 200 valores de $F y$ x 2 edificaciones). Para cada valor de f'c se estimó el módulo elástico $(E)$ con base la regresión de la Figura 2c. Así mismo la relación entre la resistencia máxima del acero y la resistencia a la fluencia del acero se mantuvo en el valor medio de los análisis estadísticos de laboratorio presentados en capítulos anteriores de tal forma que $F u / F y=1.48$. Esto implica que en realidad se variaron en cada una de las simulaciones cuatro variables principales de los materiales $\left(f^{\prime} c, E, F y y F u\right)$.
Es importante anotar que para cada combinación de valores de $f$ ' $c, E, F y$ y $F u$ (y carga axial en el caso de columnas) se calcularon 90 rótulas plásticas y 50 diagramas de interacción para cada uno de los dos edificios. Esto implica que para los análisis no lineales efectuados se determinaron $7.2 \times 10^{6}$ rótulas plásticas.

Para llevar a cabo las 80,000 simulaciones se utilizó la interfaz que tiene el programa SAP2000, CSI (2007), para manejar la mayoría de sus comandos desde la aplicación Visual Basic de Excel. Con esto se realizó una programación en la que se cambiaba el valor de $f^{\prime} c, E, F y$ y $F u$ y se guardaban los resultados en Excel. Las estadísticas y los diagramas de frecuencia de las derivas se evaluaron mediante herramientas estadísticas para ajustarle posteriormente las funciones de distribución de probabilidad más adecuadas como se hizo con las propiedades mecánicas de los materiales. Para el presente caso la deriva inelástica se define como la máxima deriva presentada en el pórtico en cada paso considerado en el análisis no lineal estático de Pushover. Con los resultados obtenidos en cada simulación se calcularon las derivas máximas para generar rótulas en nivel B, IO y LS.

\subsection{Derivas inelásticas y niveles de daño}

En la Figura 9 se presentan algunos de los 40,000 análisis de pushover efectuados sobre la edificación diseñada con el espectro de Piedemonte, incluyendo las curvas de pushover para los valores mínimos que tomaron $f^{\prime} c$ y $F y$ así como para sus respectivos valores máximos.

Por su parte en la Figura 10 y para la misma edificación (la diseñada con el espectro de Piedemonte) se presenta la deformada de la estructura para diferentes niveles de rotulación de los elementos estructurales (Niveles de fluenciaB, IO y LS). Con base en las 40,000 simulaciones para cada pórtico estudiado y usando herramientas estadísticas, se presenta en las Figuras 11 y 12 los histogramas de frecuencias de las derivas máximas para generar rótulas en niveles de desempeño de B, IO y LS. Al detenerse en las Figuras se pueden advertir agrupamientos de los datos en torno a valores medios relativamente bien definidos. Por tanto, claramente pueden definirse 
diferentes niveles de daño para la edificación en estudio.

En las Figuras 11 y 12 se presentan las funciones de distribución de probabilidad (fdp) que a la luz de las pruebas de bondad del ajuste corresponden a cada nivel de daño. Estos resultados son similares a los obtenidos en Prieto et. al. (2005) y Kircher et. al. (1997). Sin embargo, debe anotarse que según Kircher et. al. (1997), la desviación estándar de cada estado de daño, es función de la variabilidad del nivel umbral de cada estado de daño, de la variabilidad de la curva de capacidad y de la variabilidad del espectro de demanda. Esta última variable no fue considerada en el presente caso.

Las fdp de los estados de daño, Figuras 11 y 12, no son variables independientes, debido a que en la medida que se incrementa la deriva, y por lo tanto se incrementan los niveles de daño, se van generando en los elementos estructurales rotulaciones en niveles de B, IO y LS. Antes de que una estructura llegue al estado de fluencia, el comportamiento es casi elástico, en cuyo caso los elementos que presenten las mayores relaciones esfuerzos actuantes/esfuerzos resistentes tenderán a fluir. Sin embargo, una vez se generan inelasticidades del material la estructura tiende a redistribuir los esfuerzos generando niveles de daño diversos en los elementos estructurales.

En las Tabla 3 y 4 se presentan las principales características de los datos estadísticos y de las fdp ajustadas en las Figuras 11 y 12. Así mismo en la Tabla 5 se resumen los valores medios de las derivas para cada nivel de daño y los intervalos de confianza al $95 \%$ para edificios diseñados con espectro de Lacustre 500 y Piedemonte B Debe anotarse que las deriva promedio para alcanzar los

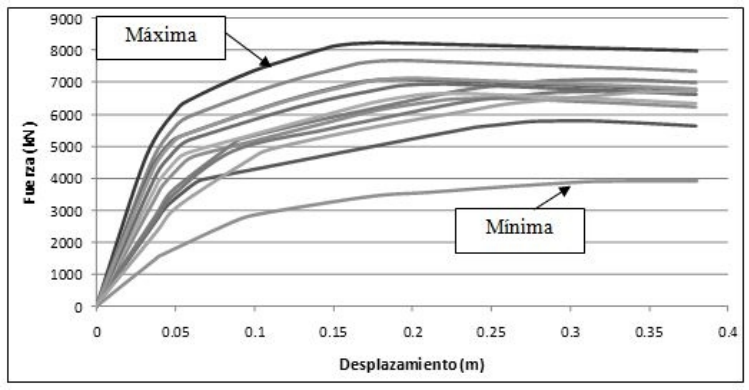

Figura 9. Curvas de capacidad

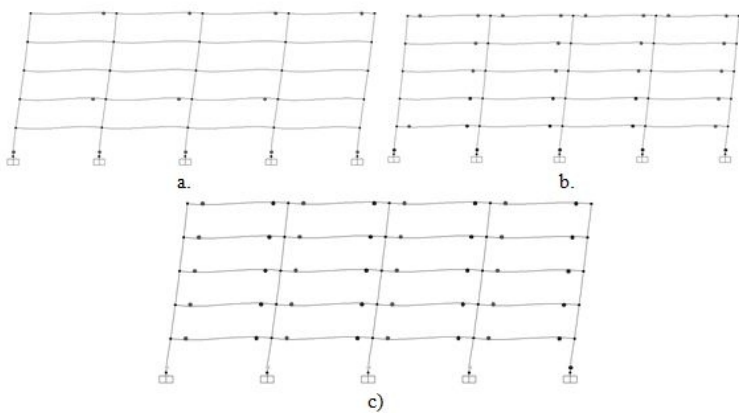

Figura 10. Elástica de la estructura en la que aparecen rótulas de a) Nivel B-fluencia b)Nivel de IO c)Nivel de LS

niveles de daño de ocupación inmediata y de seguridad a la vida obtenidas en el presente estudio están entre el $70 \%$ y el $84 \%$ de las derivas promedio indicadas en la referencia ATC40 (1996).

Un aspecto de marcada preocupación a la luz de la nueva norma sismoresistente, es que según parágrafo A.6.4.1.2 del Decreto 926 de 2010 se menciona: «Cuando se haya efectuado un análisis inelástico verificando el desempeño de la totalidad de los elementos estructurales en un rango de desempeño no mayor a "Protección de la Vida" (LS según los requerimientos del ASCE 31 y ASCE 41), las derivas pueden multiplicarse por 0.7 antes de hacer la comparación con los límites dados en la tabla A.6.4-1». En otras palabras, siguiendo la nueva Norma NSR-10, la deriva se puede volver $1.0 / 0.7=1.41 \%$ al hacer análisis inelásticos (casi el valor de 1.5\% que tenía como porcentaje de la altura de entrepiso límite de deriva el CCCSR84). Sin embargo a la luz de los resultados y considerando la variabilidad de los materiales de Bogotá, en el peor de los casos el $54 \%$ de las edificaciones que lleguen al nivel LS tendrían una deriva superior al $1.41 \%$. En este caso el parágrafo A.6.4.1.2 del Decreto 926 de 2010 no sería conservativo para preservar la vida de los habitantes de la edificación.

Aunque los anteriores límites y análisis estadísticos fueron derivados para edificaciones de 5 pisos, es claro que son un indicativo la variabilidad de las derivas inelásticas para edificaciones de concreto en Bogotá construidas con la nueva Norma NSR10 y con la microzonificación vigente. 


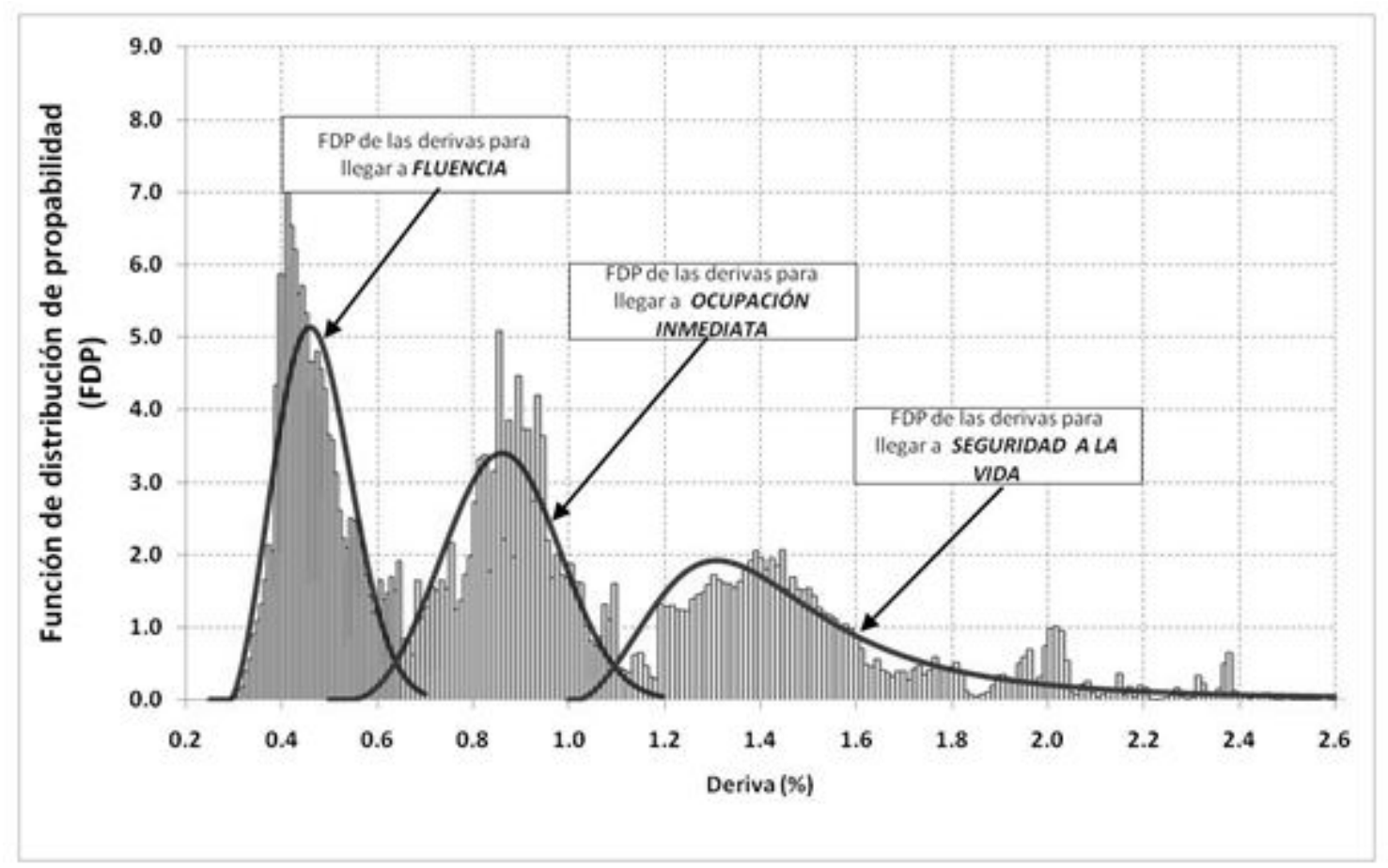

Figura 11. Funciones de distribución de probabilidad para las derivas inelásticas para edificio diseñado con espectro de Piedemonte

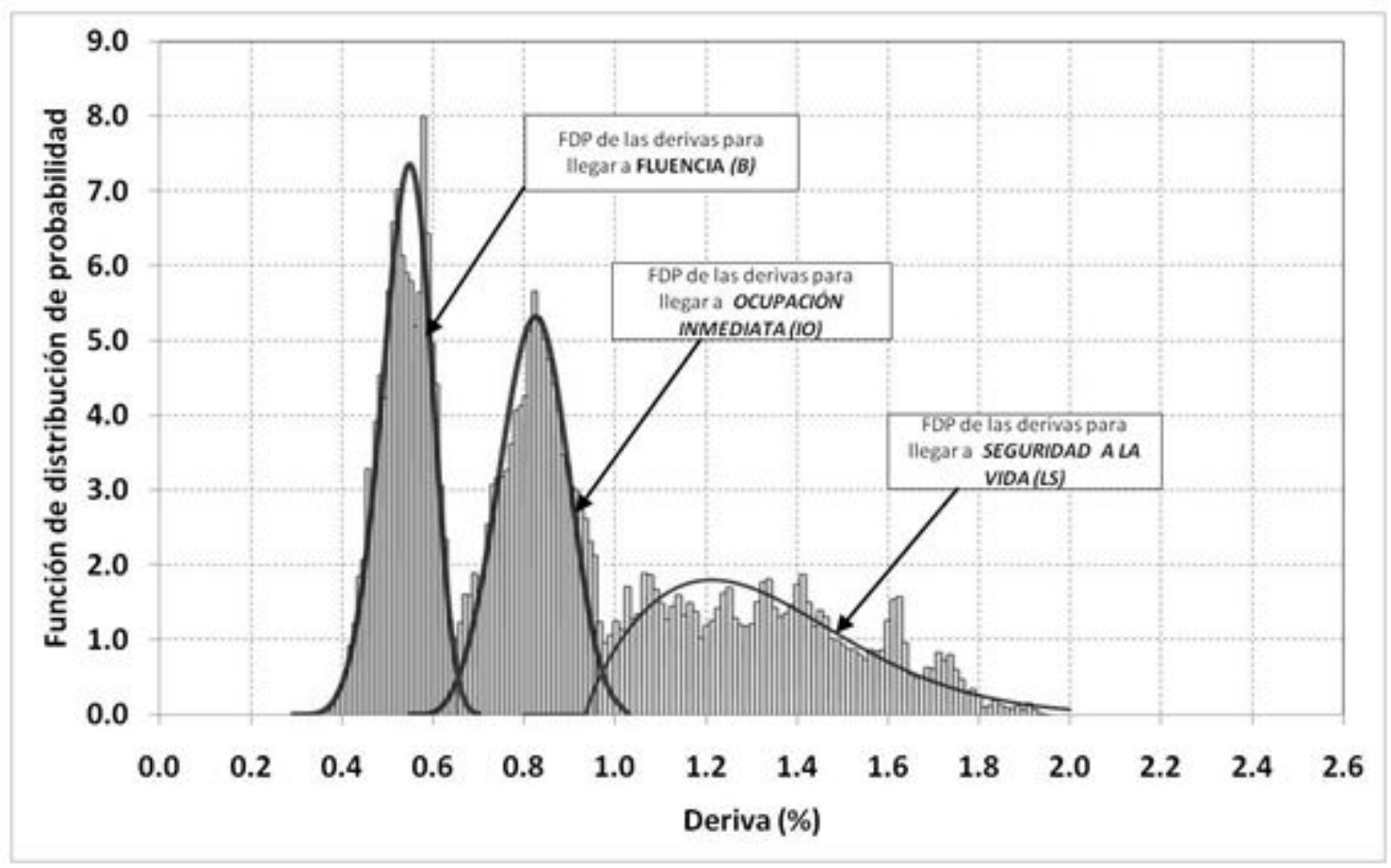

Figura 12. Funciones de distribución de probabilidad para las derivas inelásticas para edificio diseñado con el espectro de Lacustre 
Tabla 3. Datos estadísticos para las derivas que generan nivel de daño B, IO y LS para edificio diseñado con espectro de Piedemonte

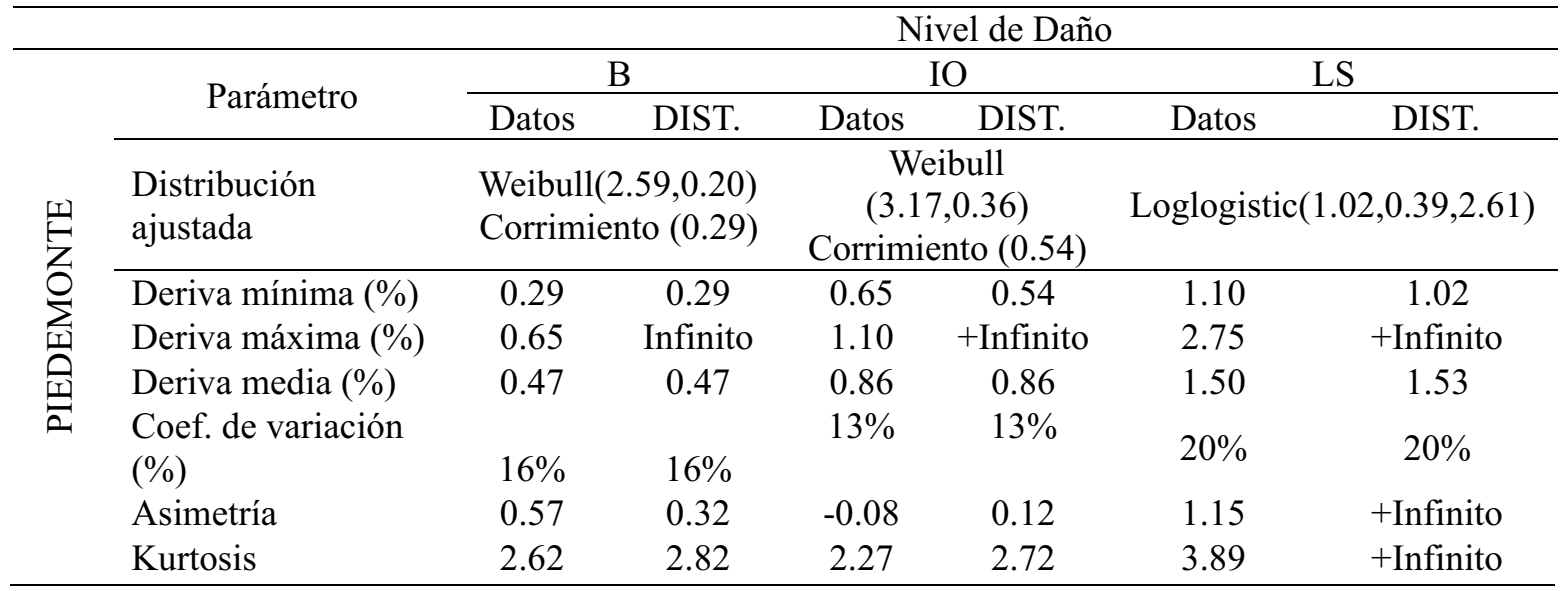

DATOS: Datos estadísticos. DIST: Distribución ajustada

Tabla 4. Datos estadísticos para las derivas que generan nivel de daño B, IO y LS para edificio diseñado con espectro de Lacustre

\begin{tabular}{|c|c|c|c|c|c|c|c|}
\hline & \multirow{3}{*}{ Parámetro } & \multicolumn{6}{|c|}{ Nivel de Daño } \\
\hline \multirow{10}{*}{ 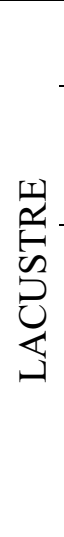 } & & \multicolumn{2}{|c|}{$\bar{B}$} & \multicolumn{2}{|c|}{$\mathrm{IO}$} & \multicolumn{2}{|c|}{ LS } \\
\hline & & Datos & DIST. & Datos & DIST. & Datos & DIST. \\
\hline & Distribución & \multirow{2}{*}{\multicolumn{2}{|c|}{$\begin{array}{l}\text { Weibull }(5.22,0.27) \\
\text { Corrimiento }(0.29)\end{array}$}} & \multirow{2}{*}{\multicolumn{2}{|c|}{$\begin{array}{l}\text { Weibull }(3.90,0.28) \\
\text { Corrimiento }(0.57)\end{array}$}} & \multirow{2}{*}{\multicolumn{2}{|c|}{$\begin{array}{l}\text { Weibull }(2.0,0.415) \\
\text { Corrimiento }(0.92)\end{array}$}} \\
\hline & ajustada & & & & & & \\
\hline & Deriva mínima (\%) & 0.37 & 0.29 & 0.66 & 0.57 & 0.96 & 0.92 \\
\hline & Deriva máxima (\%) & 0.65 & +Infinito & 0.96 & +Infinito & 1.65 & +Infinito \\
\hline & Deriva media $(\%)$ & 0.54 & 0.54 & 0.82 & 0.82 & 1.29 & 1.29 \\
\hline & $\begin{array}{l}\text { Coef. de variación } \\
(\%)\end{array}$ & $10 \%$ & $10 \%$ & $9 \%$ & $9 \%$ & $6 \%$ & $6 \%$ \\
\hline & Asimetría & -0.27 & -0.28 & -0.16 & -0.07 & 0.13 & 0.63 \\
\hline & Kurtosis & 2.44 & 2.91 & 2.25 & 2.74 & 1.92 & 3.24 \\
\hline
\end{tabular}

DATOS: Datos estadísticos. DIST: Distribución ajustada

Tabla 5. Derivas medias y e intervalos de confianza que generan nivel de daño B, IO y LS para edificios diseñados con espectro de Lacustre 500 y Piedemonte B

\begin{tabular}{|c|c|c|c|c|}
\hline \multicolumn{5}{|c|}{ Espectro de Diseño } \\
\hline & \multicolumn{2}{|r|}{ Piedemonte B } & \multicolumn{2}{|r|}{ Lacustre 500} \\
\hline Nivel de daño & Promedio & Intervalo de confianza (95\%) & Promedio & Intervalo de confianza $(95 \%)$ \\
\hline Nivel $B$ & $0.47 \%$ & $0.32 \%<$ Deriva $<0.62 \%$ & $0.54 \%$ & $0.43 \%<$ Deriva $<0.65 \%$ \\
\hline Nivel $I O$ & $0.86 \%$ & $0.64 \%<$ Deriva $<1.08 \%$ & $0.82 \%$ & $0.67 \%<$ Deriva $<0.97 \%$ \\
\hline Nivel $L S$ & $1.53 \%$ & $1.12 \%<$ Deriva $<2.60 \%$ & $1.29 \%$ & $0.99 \%<$ Deriva $<1.86 \%$ \\
\hline
\end{tabular}




\section{Conclusiones}

De acuerdo con la muestra estadística recopilada por los autores para el concreto de Bogotá, los valores de resistencia a la compresión a los 28 días, $f^{\prime} c$, varían entre $80.3 \mathrm{MPa}$ y $6.4 \mathrm{MPa}$. Así mismo y considerando el análisis estadístico realizado, la muestra analizada tiene una media de $29.2 \mathrm{MPa}$ y una desviación estándar de $11.1 \mathrm{MPa}$; y la distribución que mejor se ajusta es una LogLogístic.

Los valores del módulo de elasticidad, $E$, de la muestra oscilan entre $33,770 \mathrm{MPa}$ y $2,373 \mathrm{MPa}$. La media de la muestra es de $18,073 \mathrm{MPa}$ y la desviación estándar es de 4,149 MPa. Al igual que con la resistencia a la compresión del concreto, la distribución que mejor se ajusta es una LogLogístic.

La regresión que mejor ajusta $\sqrt{\mathrm{f}^{\prime} \mathrm{c}}$ (con $\mathrm{f}$ 'c en $\mathrm{MPa}$ ) con $E$ a la luz de los resultados de los ensayos de laboratorio es $E=3,386 \sqrt{f^{\prime} \mathrm{c}}$. Esta estimación es un 39\% inferior a la recomendada por la NSR-10, la cual sugiere usar un módulo de $\mathrm{E}=4,700 \sqrt{\mathrm{f}^{\prime} \mathrm{c}}$

A pesar de que en los desplazamientos inelásticos el módulo de elasticidad no tiene una influencia tan directa como en los desplazamientos elásticos, es claro que los desplazamientos inelásticos se ven influenciados por el desplazamiento en el que empiezan los niveles de daño de fluencia en las rótulas plásticas.

Los valores del esfuerzo de fluencia del acero, $F y$, de la muestra oscilan entre $377 \mathrm{MPa}$ y $602 \mathrm{MPa}$. La media de la muestra es de $426 \mathrm{MPa}$ y el coeficiente de variación es de $5.8 \%$. La distribución que mejor se ajusta es una LogLogístic.

Los valores del esfuerzo máximo resistente del acero, $F u$, de la muestra oscilan entre $373 \mathrm{MPa}$ y $790 \mathrm{MPa}$. La media de la muestra es de $631 \mathrm{MPa}$ y el coeficiente de variación es de $5.1 \%$. La distribución que mejor se ajusta es una Gamma.

Con base en la variabilidad del acero y el concreto, la deriva promedio para alcanzar un nivel de fluencia (B), ocupación inmediata (IO) y protección a la vida (LS) en edificaciones de 5 pisos diseñadas con la NSR 10 y con el espectro de Piedemonte B de la microzonificación sísmica de Bogotá son respectivamente: $0.47 \%, 0.86 \%$ y $1.53 \%$.

Con base en la variabilidad del acero y el concreto, la deriva promedio para alcanzar un nivel de fluencia (B), ocupación inmediata (IO) y protección a la vida (LS) en edificaciones de 5 pisos diseñadas con la NSR 10 y con el espectro de Lacustre 500 de la microzonificación sísmica de Bogotá son respectivamente: $0.54 \%, 0.86 \%$ y $1.29 \%$.

A la luz de los resultados y considerando la variabilidad del acero y el concreto en Bogotá, en el peor de los casos el $54 \%$ de las edificaciones que lleguen al nivel LS tendrían una deriva superior al 1.41\%; lo cual implica que el parágrafo A.6.4.1.2 de la NSR 10 no sería conservativo al hablar de nivel de daño $L S$.

\section{Referencias bibliográficas}

AIS (Asociación Colombiana de Ingeniería Sísmica) (2010). Reglamento colombiano de construcción sismo resistente (NSR-10). Bogotá Colombia.

AIS (Asociación Colombiana de Ingeniería Sísmica) (1998). Normas Colombianas de Diseño y Construcción Sismo Resistente (NSR-98). Bogotá Colombia.

AIS (Asociación Colombiana de Ingeniería Sísmica) (1984). Código Colombiano de Construcciones Sismo Resistentes, elaborado con base en el trabajo del Comité AIS-100 de la AIS; Decreto 1400 de 1984.

Ang, A.H.-S. (1973). Structural Risk Analysis and Reliability-Based Design. Journal of Structural Engineering Division, 99, 1973, Pag 20-35

ATC (Applied Technology Council) (1996). ATC40 (1996) Seismic Evaluation and Retrofit of Concrete Building Vol. 1. Seismic Safety Commission. California, USA. 
Canavos, George (1986). Probabilidad y estadística, aplicaciones y métodos. Mc Graw Hill.

CSI (Computers and Structures Inc). (2007). SAP 2000. Static and Dynamic Finite Element Analysis of Structures, Nonlinear.

FEMA/NIBS(1999). Earthquake Loss Estimation Methodology, Federal Emergency Management Agency. National Institute of Building Sciences. Washington D.C.

FEMA356 (2000). Federal emergency management agency. Prestandard and Commentary for the Seismic Rehabilitation of Buildings.

FOPAE (Fondo de prevención y atención de emergencias). (2010). Zonificación de la respuesta sísmica de Bogotá para el diseño sismo resistente de edificaciones. Informe final. Bogotá.

García, L. E. (1996) Economic considerations of displacement-based seismic design of structural concrete buildings. Structural Engineering International Vol $6 n^{\circ} 4$, 1669.IABSE. Zurich, Suiza.

Kircher, C.A., Nassar, A., Kustu, O. y Holmes, T. (1997) Development of Building Damage Functions for Earthquake Loss Estimation. Earthquake Spectra, 13, 4663-382.

Muñoz, E. Ruiz, D. Prieto, J. (2005). Estimación de la confiabilidad estructural de una edificación indispensable mediante análisis no lineales estáticos de pushover. Congreso Chileno de Sismología e Ingeniería Antisísmica, IX Jornadas. Artículo $N^{\circ}$ A10-13. Concepción, Chile.

NTC2289. Norma Técnica Colombiana (ICONTEC) (2005). Barras corrugadas y lisas de acero de baja aleación para refuerzo de concreto. Ensayo de tensión.

NTC4025. Norma Técnica Colombiana (ICONTEC) (2005). Concretos. Método de ensayo para determinación del módulo de elasticidad estático y la relación de Poisson en concreto a la compresión.
Prieto, J. Muñoz, E. Ruiz, D. Ramos A. (2005). Estimación de la incertidumbre aleatoria del punto de desempeño de una edificación esencial. Congreso Chileno de Sismología e Ingeniería Antisísmica, IX Jornadas. Artículo $N^{\circ}$ A04-02. Concepción, Chile.

González, V.; Botero J.C.; Rochel R.; Vidal J.; Álvalrez, M. (2005). Propiedades mecánicas del acero de refuerzo utilizado en Colombia. Revista Ingeniería y Ciencia. Volumen 1. Número 1. Pp 67 -76 .

Ruiz, D. Vacca, Hy León, M. (2007). Propuesta de modificación de la ecuación para la estimación del módulo de elasticidad del concreto en función de la resistencia a la compresión para Bogotá. Revista de la Escuela Colombiana de Ingeniería N67 Julio-Septiembre. pp 7-15.

Sánchez, Mauricio (2004). Introducción a la confiabilidad y evaluación de riesgos. Facultad de Ingeniería, Universidad de los Andes, Ediciones Uniandes. Bogotá, Colombia. 